Research Paper

\title{
Huaier Augmented the Chemotherapeutic Sensitivity of Oxaliplatin via Downregulation of YAP in Hepatocellular Carcinoma
}

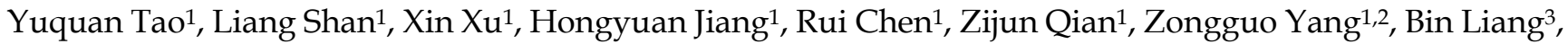 \\ Haiyin Zheng ${ }^{3}$, Feng $\mathrm{Cai}^{3}$, Yongchun $\mathrm{Yu}^{1,4}{ }^{\circledR}$, Lifang $\mathrm{Ma}^{3 凶}$ \\ 1. Shanghai Municipal Hospital of Traditional Chinese Medicine, Shanghai University of Traditional Chinese Medicine, Shanghai, 200071, P.R. China. \\ 2. Shanghai Public Health Clinical Center, Fudan University, Shanghai, 201508, P.R. China. \\ 3. Department of Clinical Laboratory Medicine, Shanghai Municipal Hospital of Traditional Chinese Medicine, Shanghai University of Traditional Chinese Medicine, \\ Shanghai, 200071, P.R. China. \\ 4. Shanghai Chest Hospital, Shanghai Jiao Tong University, Shanghai, 200030, P.R. China. \\ $\triangle$ Corresponding authors: Dr. Lifang Ma, M.D., Ph.D. No.274 Middle Zhijiang Road, Jingan District, Shanghai, P.R. China, 200071. Fax and Tel: 86-21-56634252; \\ E-mail: malifang0606118@126.com and Prof. Yongchun Yu, M.D., Ph.D. No.274 Middle Zhijiang Road, Jingan District, Shanghai, P.R. China, 200071. Fax and Tel: \\ 86-21-66313245; E-mail: yuyongchun1255@126.com
}

(c) Ivyspring International Publisher. This is an open access article distributed under the terms of the Creative Commons Attribution (CC BY-NC) license (https://creativecommons.org/licenses/by-nc/4.0/). See http://ivyspring.com/terms for full terms and conditions.

Received: 2018.03.06; Accepted: 2018.09.04; Published: 2018.10.10

\begin{abstract}
For unresectable Hepatocellular carcinoma $(\mathrm{HCC})$, chemotherapy is still an important treatment strategy. Oxaliplatin (Oxa) is an effective treatment of $\mathrm{HCC}$ after sorafenib treatment failure. However, the intrinsic or acquired resistance of Oxa affected the chemotherapeutic sensitivity. By analyzing the data of GEO Database, we found that Oxa aberrantly increased the expression of Cysteine-rich61 (Cyr61) in HCC cell lines. Subsequently, in Bel-7404 and SMMC-7721 cells after treated with Oxa, it was verified that the expression of Cyr61 and Yes-associated protein (YAP) was increased. Moreover, we found that blockade of YAP promoted Oxa-induced cell apoptosis for the first time. Meanwhile, our previous study demonstrated that Huaier $(\mathrm{HE})$ inhibited the expression of YAP. Further study found that combination treatment of Oxa and HE had a significantly synergistic anti-cancer effect and significantly inhibited the expression of YAP and apoptosis related proteins. Taken together, we have observed that overexpression of YAP significantly reduced the chemotherapeutic sensitivity of Oxa in HCC for the first time. Combination treatment of Oxa and HE solved this problem.
\end{abstract}

Key words: YAP, chemotherapeutic sensitivity, oxaliplatin, Huaier, synergistic effect, Hepatocellular carcinoma

\section{Introduction}

Hepatocellular carcinoma is one of the common malignancies, which has high mortality rate and seriously jeopardizes the health of human in the world [1]. Many patients with advanced HCC are unfit for surgery. Hence, systemic comprehensive treatment is still an important treatment for unresectable HCC [2]. Sorafenib remains the optimal treatment for advanced HCC by current data. However, there is lacking of unified treatment strategy after sorafenib treatment failure [3].

Oxaliplatin is the third generation platinum drugs and displays the anti-tumor effect in many tumors containing HCC [4]. A multicenter phase III clinical study (EACH test) showed that the treatment of FOLFOX4 (Oxa, fluorouracil and leucovorin) protocol prolonged time to tumor progression (TTP) and overall survival (OS)[5]. But the intrinsic or acquired resistance of Oxa affected the chemotherapeutic sensitivity [6]. Therefore, the molecular mechanism about the chemotherapeutic sensitivity of Oxa in HCC is worthy of further study.

Previous data of gene expression profiles (GSE51951) showed that Oxa aberrantly increased the expression of Cyr61 [7]. However, the expression of Cyr61 commonly promoted recurrence and metastasis of HCC. Cyr61 and the same gene family member 
Connective Tissue Growth Factor (CTGF) are mainly regulated by YAP in Hippo signaling pathway [8, 9]. Yes-associated protein is a pivotal transcriptional co-activator of Hippo signaling pathway [10]. In HCC, YAP located in cell nucleus promotes the expression of downstream genes (including Cyr61, CTGF and so on), which is related to tumorigenesis, cell proliferation, apoptosis and migration[11]. Although previous studies have reported overexpression of YAP was related to doxorubicin resistance in HCC and Taxol, cisplatin, and 5-fluorouracil resistance in other cancers[12], none of the researches have studied if YAP expression affects the curative effect of Oxa in HCC. Therefore, in our study, we investigated the relevance between the chemotherapeutic sensitivity of Oxa and the expression of YAP in HCC cell lines.

Hence, the expression of Cyr61 and Yes-associated protein (YAP) were verified in Bel-7404 and SMMC-7721 cells after treated with Oxa. Then, we compared the expression levels of YAP and apoptosis related proteins in Bel-7404 cells and Bel-7404 YAPsh cells after treated with Oxa. The results demonstrated that the high level of YAP expression inhibited cell apoptosis induced by Oxa. Moreover, HE, also called Trametes robiniophila Murr, is a traditional medical fungus, which has been widely used as an adjuvant therapy in advanced HCC in China. Proteoglycans, containing $41.53 \%$ polysaccharides, $12.93 \%$ amino acids and $8.72 \%$ water, are the mainly active fraction of HE extracts[13]. Our previous study showed that HE inhibited the expression of YAP in HCC cell lines [14]. Therefore, further study was performed to investigate the anti-cancer effect of Oxa combination with HE in HCC and the underlying molecular mechanisms.

\section{Materials and Methods}

\section{Cell culture and vectors}

Two HCC cell lines (Bel-7404 and SMMC-7721) were obtained from the previously frozen cells of our research teams. Cell lines were cultured under standard condition containing DMEM (Hyclone, Beijing, China) medium supplemented with $10 \%$ fetal bovine serum (Gibco, USA), $1 \%$ penicillin-streptomycin solution (KeyGen, KGY0023). The shRNA of YAP were obtained from previous studies $[15,16]$.

\section{Preparation of HE and Oxa}

Huaier was purchased from Qidong Gaitianli Pharmaceutical Co., Ltd. (Jiangsu, China). Huaier was dissolved in phosphate-buffered saline (PBS; KeyGen, Nanjing, China) at a concentration of $500 \mathrm{mg} / \mathrm{ml}$ and stored at $4^{\circ} \mathrm{C}$ before use. Oxaliplatin (Oxa; Sigma, O9512) was dissolved in dimethyl sulfoxide (DMSO; Sigma, D4540) at a concentration of $1 \mathrm{mg} / \mathrm{ml}$ and stored at $-20^{\circ} \mathrm{C}$ before use. HE and Oxa solution were filtered through $0.22 \mu \mathrm{m}$ filter before storage and diluted to the proper concentration before each experiment.

\section{Cell proliferation assay and the synergistic effect}

The proliferation activities of Bel-7404 and SMMC-7721 cells were detected by cell counting kit-8 (CCK8; Beyotime Biotechnology Inc., Shanghai, China). Specifically, 5000 cells/ well were seeded onto 96-well plates. After $12 \mathrm{~h}$, cells were treated with HE, Oxa, or Oxa combined with HE at different concentrations for 24 h. $10 \%$ CKK8 reagent was added and incubated for $1 \mathrm{~h}$. The OD value at a wavelength of $450 \mathrm{~nm}$ was measured by a microplate reader (Thermo scientific, Waltham, MA, USA). The combination index $(\mathrm{CI})$ value represents the mutual effect of drugs and was calculated by calcusyn 2.0 software. $\mathrm{CI}<1.0$, represents synergistic effect. $\mathrm{CI}=1$, indicates additive effects; $\mathrm{CI}>1$, denotes antagonistic effect.

\section{Clone formation assay}

Bel-7404 and SMMC-7721 cells (1000 cells per well) were seeded onto 12-well plates and cultured for $24 \mathrm{~h}$. Cells were treated with HE, Oxa, or Oxa combined with HE for 6 days. The culture medium was replaced every 3 days and the treatment was maintained. Cells were fixed with precooled 95\% ethanol and then stained with $0.1 \%$ crystal violet (Sigma, V5265).

\section{Flow Cytometry analysis of cell apoptosis and cell cycle}

An Annexin V-FITC/PI apoptosis detection kit (KeyGen, Nanjing, China) was used to distinguish the apoptotic cells. Cells were collected and washed with precooled PBS. Then, $10^{5}$ cells were suspended with $500 \mu \mathrm{l}$ Annexin V Binding Buffer and incubated with Annexin V-FITC and propidium iodide (PI) solution in dark place. After 10 minutes, cells were detected by flow cytometry within $1 \mathrm{~h}$. The FlowJo V10 software was used to analyze apoptosis results.

Cell cycle was detected by PI detection kit (KeyGen, Nanjing, China). After starved for 24 h, cells were treated with different drugs (HE, Oxa, or Oxa combined with HE) for $24 \mathrm{~h}$. Then cells were collected and the concentration was adjusted to $1^{*} 10^{6} / \mathrm{ml}$. Cells were fixed with precooled $70 \%$ ethanol for $12 \mathrm{~h}$ and heated with RNase in water bath at $37^{\circ} \mathrm{C}$ for 30 minutes. PI was added to label cell nucleus DNA at $4^{\circ} \mathrm{C}$. After 30 minutes, cells were detected by flow cytometry within $1 \mathrm{~h}$. The ModFit LT 5.0 software was used to analyze cell cycle results. 
Western blotting (WB) and

Immunofluorescence (IF)

For WB, cells were treated with different drugs (HE, Oxa, or Oxa combined with HE) for $24 \mathrm{~h}$. At $4{ }^{\circ} \mathrm{C}$, cells were lysed by cell lysis buffer for Western and IP (KeyGen, KGP701) for $1 \mathrm{~h}$ and then centrifuged at 12, $000 \mathrm{~g}$ for $10 \mathrm{~min}$. We collected the supernatants and tested total proteins concentration using BCA Protein Assay Kit (KeyGen, KGP902). The separation of total proteins was through electrophoresis using SDS-PAGE gels and then followed the standard WB. The primary antibodies were listed as follows: $\beta$-actin (ImmunoWay, YM3028) YAP (Abcam, ab52771), P-YAP (Abcam, ab76252), Bcl2 (ImmunoWay, YM3 041), Cyclin D1 (Abcam, ab134175), Active-Caspase3 (Abcam, ab32042), Cleaved Caspase Substrate (CST, \#8698), Cyr61 (ImmunoWay, YN2003), CTGF (Santa cruz, sc-373936), $\beta$-catenin (Abcam, ab32572), $\beta$-TrCP (CST, \#4394). The secondary antibodies were purchased from cell signaling technology (\#7074 and \#7076). The blots were visualized by ECL (KeyGen, KGP1128).

For IF, cells were fixed with $4 \%$ paraformaldehyde (DingGuo, AR-0211) for $15 \mathrm{~min}$ and washed with PBS for three times (5 min per time). Cells were incubated with 200 $\mu$ l PBS solution containing 3\% FBS, $1 \%$ Goat serum and $0.1 \%$ Triton X-100 for 2 hours at room temperature. Next, cells were incubated with YAP antibody (Abcam, ab52771) overnight at $4^{\circ} \mathrm{C}$. Similarly, cells were washed with PBS for three times (5 min per time) before incubation with $\mathrm{Cy} 3$ conjugated secondary antibodies (Google Biotechnology, GB21303). DAPI was used to stain cell nucleus. The Crest Optics X-Light V2 spinning disk confocal microscope was used to analyze the results.

\section{Quantitative real-time PCR (qRT-PCR)}

Total RNA was extracted from cells using Trizol reagent (Sigma, T9424) and reversed transcription was performed using cDNA Synthesis SuperMix (Biotool, B24403). The mRNA expression level was detected using SYBR Green Mix (Biotool, B21202). Data were analyzed using the $\triangle \triangle \mathrm{Ct}$ method. The primer sequences were listed in Supplementary Table 1.

\section{GEO Database analysis}

Raw data were download from GEO Database (GSE51951). Differential genes expression analysis was performed with the R limma software package. Genes with fold changes $>2$ and $p$ value $<0.05$ are selected as the significantly differential genes. Fifty significantly differential genes were chosen to draw the heatmap using the R pheatmap software package.

\section{Statistical analysis}

All data were analyzed by the SPSS 21.0 software (SPSS lnc. Chicago, USA) and shown by the mean \pm standard deviation (SD) for three independent experiments. The Student t-test and one-way ANOVA were used to test the differences of measurement data. Count data were analyzed using chi-square test or non-parameter test. $P$ values $<0.05$ were considered statistically significant.

\section{Results}

\section{Effects of Oxa to HCC cell lines on cell apoptosis and cell cycle}

In order to determinate the $50 \%$ inhibitive concentration (IC50) value of Oxa, Bel-7404 and SMMC-7721 cells were treated with different concentrations of Oxa. The cell viability was significantly inhibited by Oxa at a concentration of $10 \mu \mathrm{g} / \mathrm{ml}$ and the cell inhibitory rate was 50\%. In addition, Bel-7404 and SMMC-7721 had similar IC50 value (Fig. 1A). Because oxaliplatin was dissolved in $5 \%$ glucose injection in clinical, CCK8 assay was also performed to detect the influence on the cell viability when oxaliplatin was dissolved in different solvents. The results showed that DMSO did not affect the function of oxaliplatin on cell viability (Supplementary Fig. 1A).

Next, we examined whether Oxa regulated cell apoptosis and cell cycle at a concentration of 10 $\mu \mathrm{g} / \mathrm{ml}$. The results revealed that Oxa induced cell cycle arrest at the S phase (Fig. 1B). However, Oxa did not significantly induce cell apoptosis (Fig. 1C). WB analyses were consistent with the flow cytometry (FCM) results. The cell cycle checkpoint protein CyclinD1 was significantly decreased, but the apoptosis related proteins (Bcl-2, Active Caspase3, and Cleaved Caspase Substrate) did not show significant change (Fig. 1D).

\section{The increased expression of YAP inhibited the apoptosis induced by Oxa in HCC cell lines}

Based on the reported above, we therefore investigated why Oxa did not significantly induce cell apoptosis in HCC cell lines. Previous studies have detected gene expression profiles of tumor tissues from Oxa-treated and 5\% GS-treated (control) [7]. We screened 348 differential genes (50 genes for heatmap) with fold changes $>2$ and $p$ value $<0.05$ and found that the expression of Cyr61 was aberrantly elevated (Fig. 2A). Therefore, further study was performed to identify the expression levels of proteins including phosphorylated YAP (P-YAP), YAP, Cyr61, $\beta$-catenin, and CTGF. WB analysis demonstrated that the expression of YAP and Cyr61 were indeed increased 
in Bel-7404 and SMMC-7721 cells after treated with Oxa (Fig. 2B and Supplementary Fig. 1B). Oxa also decreased the expression of P-YAP. In addition, the mRNA expression levels of YAP, CTGF, Cyr61, ANKRD1, and $\beta$-catenin were also verified in Bel-7404 and SMMC-7721 cells. Consistent with the WB results, Oxa promoted the expression of YAP, CTGF, and Cyr61 (Fig. 2C). Moreover, Oxa reduced the expression of YAP located in cytoplasm (Fig. 2D). As reported above, we further verified the relationship between the expression of YAP and the apoptosis induced by Oxa. FCM and WB analysis showed that Oxa-induced cell apoptosis was augmented while YAP was knocked down (Fig. 2E, F). These results showed that the expression of YAP was related to the apoptosis induced by Oxa in Bel-7404 and SMMC-7721 cell lines.

\section{Huaier potentiated the cytotoxicity of Oxa in a synergistic manner}

Our previous studies showed that HE inhibited the expression of YAP in HCC cell lines [14]. The IC50 value of $\mathrm{HE}$ was determinated and the cell viability
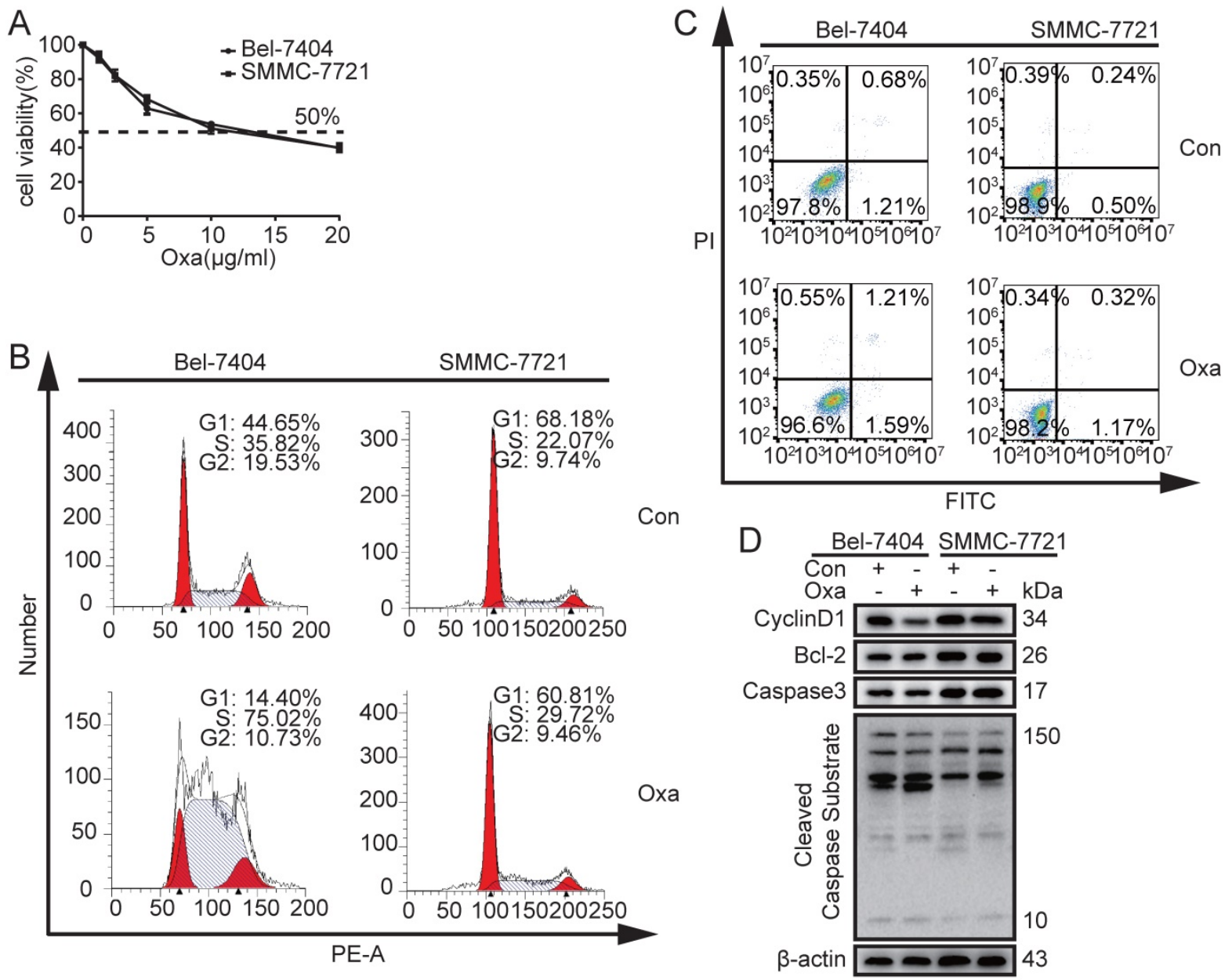

Fig. 1. Effects of Oxa to Bel-7404 and SMMC-7721 cells on cell apoptosis and cell cycle. (A) Cell viability was measured by CCK8 assay and $50 \%$ inhibitive concentration (IC50) of Oxa was calculated also. (B-D) Bel-7404 and SMMC-7721 cells were incubated with Oxa (10 $\mu \mathrm{g} / \mathrm{ml})$ for $24 \mathrm{~h}$. (B) HCC cell cycle were examined by flow cytometry (FCM) following PI staining. (C) Early apoptotic cells were detected by FCM following Annexin V-FITC/PI staining. (D) WB assay analyzed the protein expression of Cyclin DI, Bcl-2, Active Caspase3, and Cleaved Caspase Substrate. $\beta$-actin was used as a loading control. Representative results of three experiments were shown.

was reduced to a level of $50 \%$ when treated with HE at a concentration of $12 \mathrm{mg} / \mathrm{ml}$ (Supplementary Fig. 1C). Consequently, we investigated if the combination treatment of HE $(12 \mathrm{mg} / \mathrm{ml})$ and Oxa $(10 \mu \mathrm{g} / \mathrm{ml})$ promotes cell apoptosis. Significant morphological changes were observed after combination treatment by inverted microscope (Fig. 3A). The clone formation capacity was significantly inhibited by the combination treatment (Fig. 3B). In Bel-7404 cells, the combination treatment also promoted cell early apoptosis (Fig. 3C). Then, the combination effects of $\mathrm{HE}$ and Oxa was detected by CCK-8 assay. Compared to Oxa alone, combination treatment (HE: Oxa at 12 $\mathrm{mg} / \mathrm{ml}: 10 \mu \mathrm{g} / \mathrm{ml}$ ) significantly augmented the inhibitory effect. In order to determinate whether the combination effect of HE with Oxa was additive or synergistic, the combination index $(\mathrm{CI})$ values were calculated using calcusyn 2.0 software. The $\mathrm{CI}$ value represents the mutual effect of drugs and $\mathrm{CI}<1.0$ indicates synergistic effect, $\mathrm{CI}=1$ suggests additive effects, CI >1 denotes antagonistic effect (Fig. 3D). 

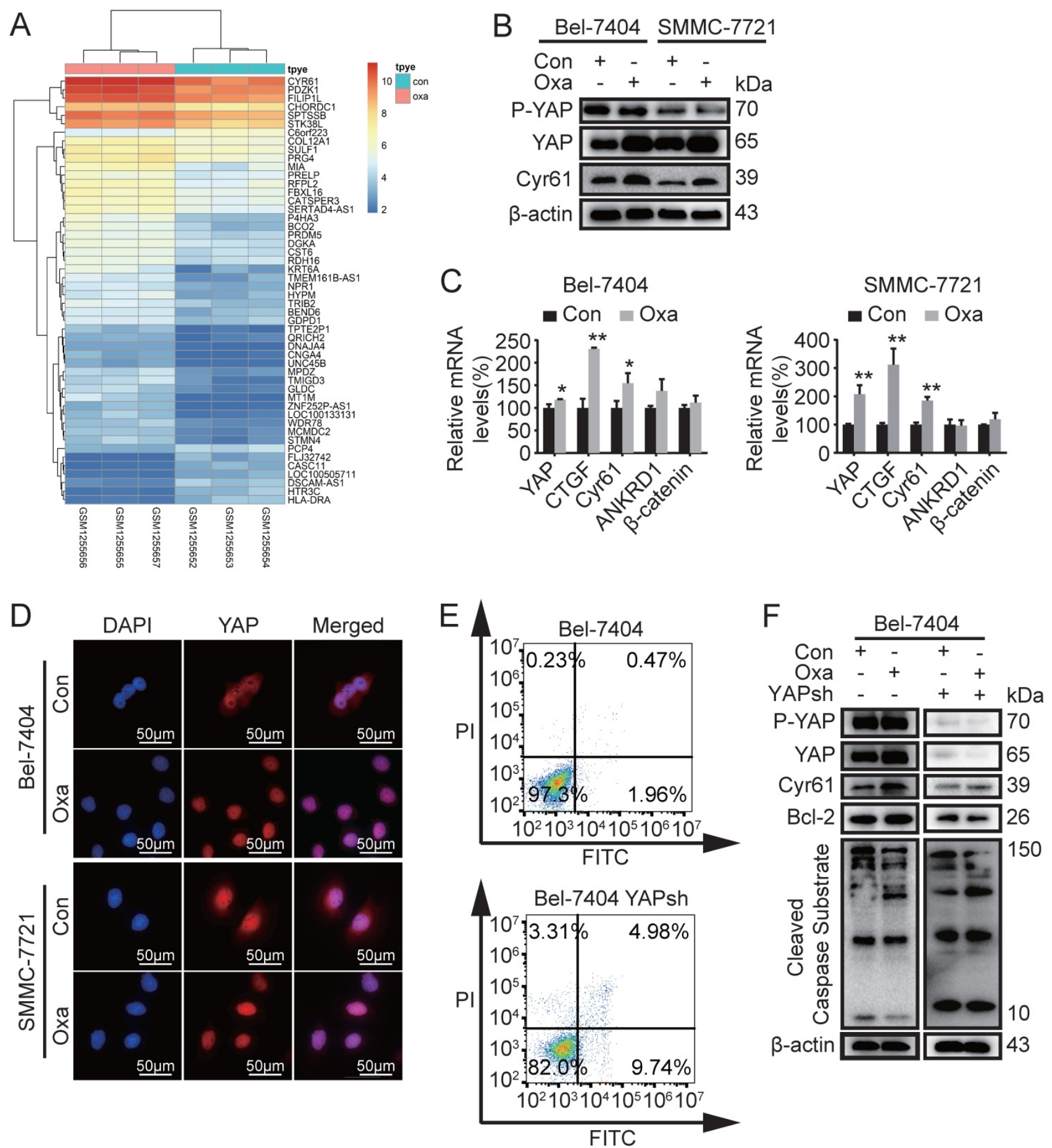

Fig. 2. Oxaliplatin induced the expression of Cyr61, YAP and apoptosis related genes. (A) Differential expression genes of tumor tissues were screened between Oxa-treated and 5\% GS-treated (control). (B-D) Bel-7404 and SMMC-7721 cells were incubated with Oxa (10 $\mu \mathrm{g} / \mathrm{ml})$ for $24 \mathrm{~h}$. (B) The expression levels of phosphorylated YAP (P-YAP), YAP, and Cyr61 were assayed by WB analysis. (C) Relative mRNA levels of YAP, CTGF, Cyr61, ANKRD1, and $\beta$-catenin were detected by quantitative RT-PCR analysis. Each bar represented means \pm SD from three independent experiments. $*, P<0.05, * *, P<0.01$ as analyzed by one-way ANOVA. (D) Oxaliplatin affected the intracellular location of YAP, which were examined by IF assay. (E) Early apoptotic cells were detected by FCM following Annexin V-FITC/PI staining. (F) WB assay detected the expression levels of P-YAP, YAP, Cyr61, Bcl2, and Cleaved Caspase Substrate in Bel-7404 and Bel-7404 YAPsh cells after treated with Oxa (10 $\mathrm{Mg} / \mathrm{ml})$ for $24 \mathrm{~h}$. Representative results of three experiments were shown.

\section{Huaier promoted Oxa-induced cell apoptosis by inhibiting the expression of YAP}

As reported above, downregulation of YAP protein expression promoted Oxa-induced cell apoptosis. HE induced apoptosis of HCC cell lines by inhibiting the expression of YAP. Moreover, HE augmented Oxa-induced cell apoptosis in a synergistic manner. Therefore, we further investigated whether HE promotes Oxa-induced cell apoptosis by suppressing the expression level of YAP. The results 
revealed that blockade of YAP significantly reduced cell viability of Bel-7404 cells after treated with HE, Oxa, and Oxa combined with HE (Fig. 4A). Compared with negative control and Oxa group, the combination treatment significantly decreased the protein expression levels of YAP, Cyr61, CyclinD1, Bcl2, and $\beta$-catenin, and also increased the expression of P-YAP and Cleaved Caspase Substrate in Bel-7404 and SMMC-7721 cells. The protein expression level of $\beta$-TrCP were not significant changes in Bel-7404 cells when treated with HE, Oxa, and Oxa combined with HE (Fig. 4B, C and Supplementary Fig. 1D). At the same time, the expression levels of above proteins were detected in Bel-7404 YAPsh cells. The concentration of drugs and the total proteins between Bel-7404 cells and Bel-7404 YAPsh cells were consistent. WB analysis showed that P-YAP and Cyr61 was significantly inhibited because of the blockade of YAP. Compared with negative control and Oxa group in YAPsh Bel-7404 cells, the combination treatment also decreased the protein expression levels of YAP, Cyr61, CyclinD1, and Bc12, and also increased the expression of P-YAP and Cleaved Caspase Substrate. Furthermore, compared with the corresponding proteins expression in Bel-7404 cells, the expression levels of Cyr61, CyclinD1, and Bcl-2 were significantly decreased in Oxa group and combination group, and the expression level of Cleaved Caspase Substrate were significantly increased (Fig. 4B). In addition, the relative expression level of P-YAP was calculated according to the gray value of corresponding blots. The results showed that the P-YAP protein expression level of combination treatment group was higher than negative control and Oxa group, and the differences were statistically significant. Interestingly, compared with HE group, the changes of P-YAP in combination group were inconsistent between Bel-7404 cells and SMMC-7721 cells (Fig. 4D). Taken together, these results demonstrated that HE promoted Oxa-induced cell apoptosis by suppressing the expression of YAP (Fig. 4E).

\section{Discussion}

Yes-associated protein, which is a pivotal transcriptional coactivator of Hippo signaling pathway, promotes the transcription of downstream genes (including Cyr61, CTGF and so on) and thus plays important roles in tumorigenesis, cell proliferation, apoptosis, migration[10, 11]. Although previous studies have reported overexpression of YAP was related to the chemotherapeutic sensitivity of doxorubicin, Taxol, cisplatin, and 5-fluorouracil [12], none of the researches have studied if YAP expression affects the chemotherapeutic sensitivity of
Oxa in HCC. In this study, Oxa cannot significantly induced cell apoptosis in HCC cell lines (Fig. 1C, D). Interestingly, Oxa aberrantly promoted the expression of YAP in HCC cell lines (Fig. 2A-C). Moreover, we uncovered that the expression level of YAP was related to the chemotherapeutic sensitivity of Oxa in HCC for the first time. Blockade of YAP increased the Oxa-induced cell apoptosis (Fig. 2E, F). A previous study reported that silenced Fat 4 led to the nuclear accumulation of YAP and the Fat 4 silenced cells had less chemotherapeutic sensitivity of Oxa in gastric cancer cells [17]. Similarly, Oxa had limited curative effect in treating late stage AKT/YAPS127A intrahepatic cholangiocarcinoma [18]. Although YAP was not the main research molecule in these studies, the reported data also provided evidence that the expression level of YAP probably affected the chemotherapeutic sensitivity of Oxa.

Recently, the combination usage of natural products from medicinal plants and conventional chemotherapy drugs has received lots of attention in cancer treatment. Our previous study demonstrated HE downregulated the expression of YAP in HCC cell lines [14]. In the current study, we observed that the combination treatment of Oxa and $\mathrm{HE}$ had a significantly synergistic anti-cancer effect in HCC cells for the first time (Fig. 3D). Furthermore, combination treatment of Oxa and HE significantly induced the expression of apoptosis related proteins. Compared with Bel-7404 cells, Oxa or Oxa combined with HE induced more cell apoptosis in Bel-7404 YAPsh cells, which suggested that HE enhanced Oxa-induced cell apoptosis by inhibiting the expression of YAP (Fig. 4B). Oxymatrine or Lentinus edodes polysaccharide also enhanced the anti-cancer effect of Oxa in a synergistic manner [4, 19], which suggested appropriate natural products from medicinal plants were benefited to improve the chemotherapeutic sensitivity and reduce side effects of conventional chemotherapy drugs.

It should be noted that our results were just demonstrated in two HCC cell lines. Additional experiments in vivo are warranted to verify and expand on these findings. Moreover, our study highlights the synergistic mechanism of Oxa in combination with HE in HCC. It still needs further research to illuminate the detailed mechanism of how Oxa and HE transfer signals to YAP located in cytoplasm. Now that we have demonstrated the expression of YAP is related to the chemotherapeutic sensitivity of Oxa in HCC, future studies may focus on the possible relationships between YAP and the intrinsic or acquired resistance of Oxa, which is beneficial to evaluate the sensitive of individual cancer patients to Oxa. 
A

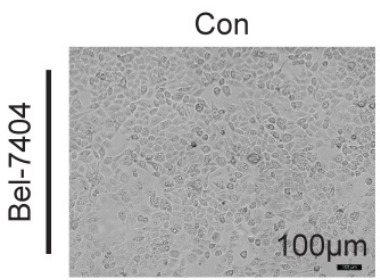

HE

Oxa

$\mathrm{Oxa}+\mathrm{HE}$
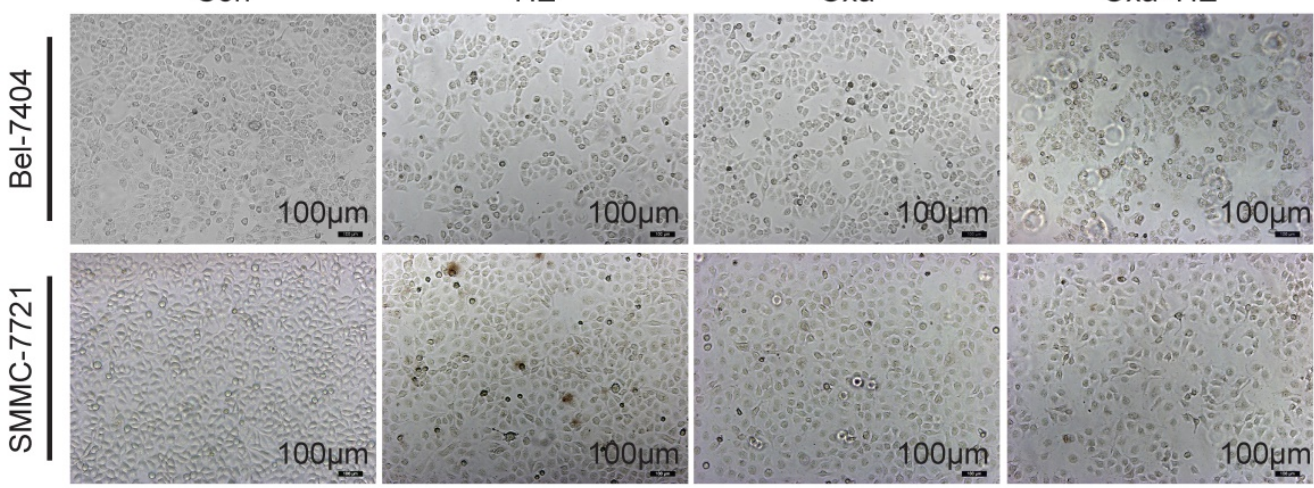

B
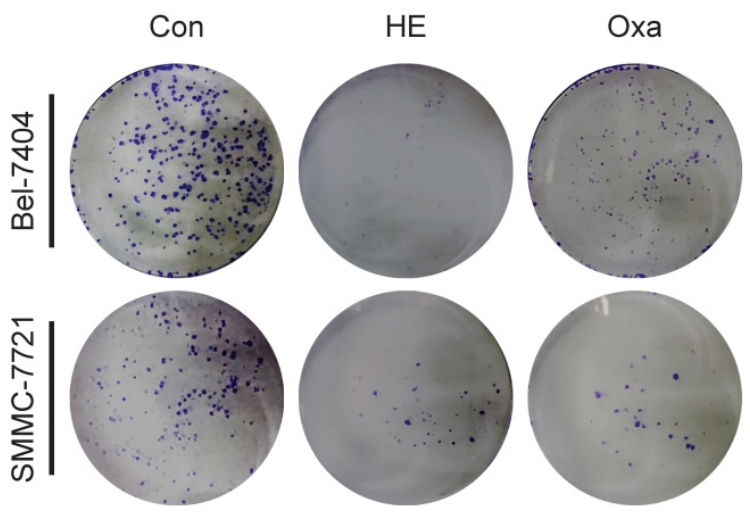

$\mathrm{Oxa}+\mathrm{HE}$

C

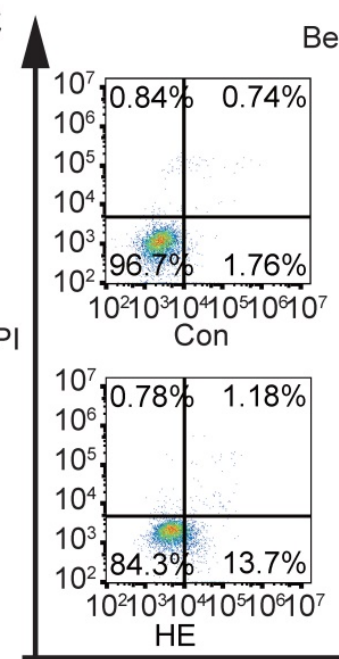

Bel-7404
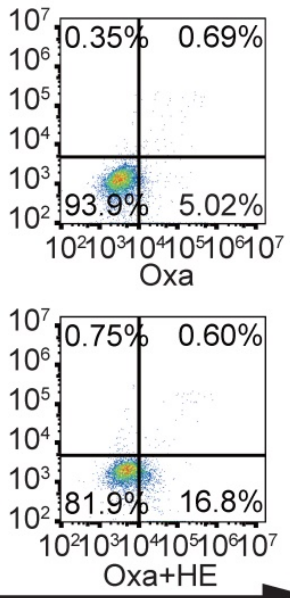

FITC
D
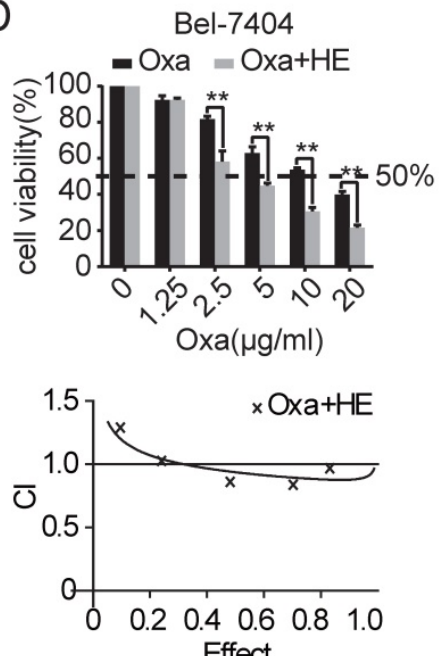

SMMC-7721
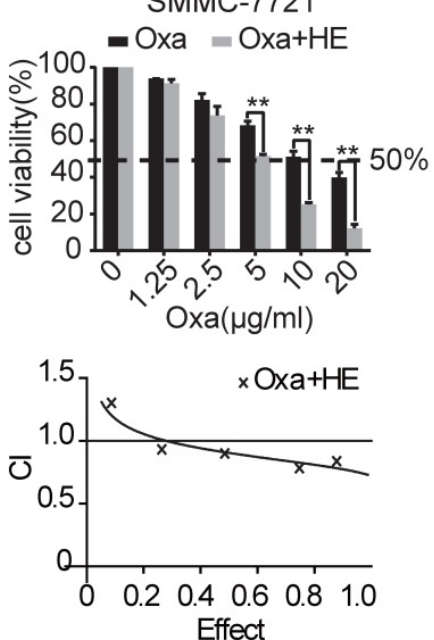

Fig. 3. Effects of Oxa, HE, and Oxa combined with HE in HCC cell lines. (A-D) Bel-7404 and SMMC-7721 cells were incubated with Oxa ( $10 \mu g / \mathrm{ml})$, HE ( $12 \mathrm{mg} / \mathrm{ml})$, or Oxa $(10 \mu \mathrm{g} / \mathrm{ml})$ combined with HE $(12 \mathrm{mg} / \mathrm{ml})$ for $24 \mathrm{~h}$. The IC50 of HE was measured by CCK 8 assay (Figure S1). (A) The morphological changes were observed by inverted microscope. (B) Clone formation capacity of Bel-7404 and SMMC-7721 cells was assessed by the clone formation assay. Cells were stained with $0.1 \%$ crystal violet after fixed. (C) Representative early apoptotic cells were detected by FCM following Annexin V-FITC/PI staining in Bel-7404 cells. The lower right quadrant of the cross represented early apoptotic cells (\%). (D) CCK-8 was performed to assess the cytotoxic effect of Oxa, HE, and Oxa combined with $\mathrm{HE}$. The combination index (Cl) value represented the mutual effect of Oxa and HE. Cl lower than 1.0 represented synergistic effect. Each bar represented means \pm SD from three independent experiments. *, $P<0.05$, **, P<0.01 as analyzed by Student $\mathrm{t}$ test. 

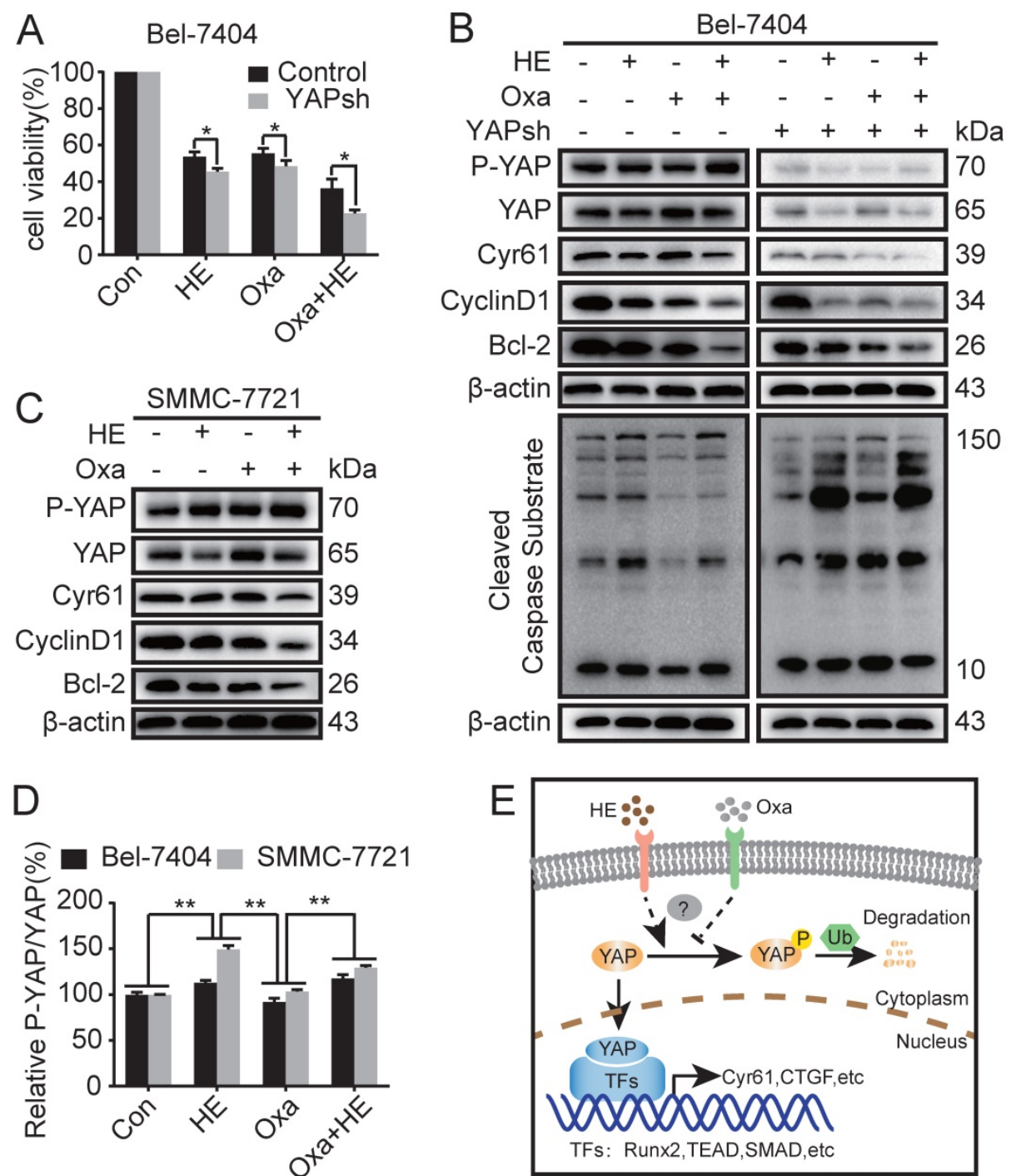

Fig. 4. Huaier enhanced Oxa-induced cell apoptosis by inhibiting the expression of YAP. (A-C) Bel-7404, Bel-7404 YAPsh, and SMMC-7721 cells were incubated with Oxa $(10 \mu \mathrm{g} / \mathrm{ml}), \mathrm{HE}(12 \mathrm{mg} / \mathrm{ml})$, or Oxa $(10 \mu \mathrm{g} / \mathrm{ml})$ combined with HE $(12 \mathrm{mg} / \mathrm{ml})$ for $24 \mathrm{~h}$. (A) Cell viability was examined by CCK-8 assay in Bel-7404 and Bel-7404 YAPsh cells. *, P<0.05 as analyzed by Student t test. (B-C) WB assay was performed to detect the protein expression of P-YAP, YAP, Cyr61, CyclinD1, Bcl-2, and Cleaved Caspase Substrate. Representative results of three experiments were shown. (D) The expression level of P-YAP was normalized to the total YAP. Each bar represented means \pm SD from three independent experiments. **, $\mathrm{P}<0.01$ as analyzed by one-way ANOVA. (E) The underlying interaction mechanism between $\mathrm{HE}$ and $\mathrm{Oxa}$ in the regulation of $\mathrm{YAP}$ expression in HCC cell lines.

\section{Supplementary Material}

Supplementary figures and tables.

http://www.jcancer.org/v09p3962s1.pdf

\section{Acknowledgements}

The authors are grateful to $\mathrm{Yu}$ Guan, Ning Li, and Chunmei Qian for technical assistance.

\section{Funding Source}

This study was supported by Natural science foundation of China (Grants 81472124 and 81774291), Talent introduction project of Shanghai Municipal Hospital of Traditional Chinese Medicine (Grant 20160501, to Lifang Ma), "Chen Guang" project supported by Shanghai Municipal Education
Commission and Shanghai Education Development Foundation (Grant 17CG43, to Lifang Ma), Innovation project of Shanghai University of Traditional Chinese Medicine (Grants JXDXSCXJH10 and JXDXSCXJH15, to Liang Shan and Yuquan Tao), and Shanghai Sailing Program (18YF1421800).

\section{Author Contributions}

Y.Y. and L.M. conceived and supervised the study; Y.T., Y.Y., and L.M. designed experiments; Y.T. and L.S. carried out the GEO Database analysis; Y.T., X.X., and H.J. performed experiments; Y.T., R.C., and Z.Q. analyzed data; Z.Y., B.L., and L.M. provided new tools and reagents; H.Z. and F.C. performed the statistical analysis; Y.T. wrote the manuscript; All authors read and approved the final manuscript. 


\section{Competing Interests}

The authors have declared that no competing interest exists.

\section{References}

1. Siegel RL, Miller KD, Jemal A. Cancer Statistics, 2017. CA Cancer J Clin. 2017; 67: 7-30.

2. Finn RS, Zhu AX, Farah W, Almasri J, Zaiem F, Prokop LJ, et al. Therapies for advanced stage hepatocellular carcinoma with macrovascular invasion or metastatic disease: A systematic review and meta-analysis. Hepatology. 2018; 67: 422-35.

3. Niu L, Liu L, Yang S, Ren J, Lai PBS, Chen GG. New insights into sorafenib resistance in hepatocellular carcinoma: Responsible mechanisms and promising strategies. Biochim Biophys Acta. 2017; 1868: 564-70.

4. Zhang Y, Li Q, Wang J, Cheng F, Huang X, Cheng Y, et al. Polysaccharide from Lentinus edodes combined with oxaliplatin possesses the synergy and attenuation effect in hepatocellular carcinoma. Cancer Lett. 2016; 377: 117-25.

5. Qin S, Bai Y, Lim HY, Thongprasert S, Chao Y, Fan J, et al. Randomized, multicenter, open-label study of oxaliplatin plus fluorouracil/leucovorin versus doxorubicin as palliative chemotherapy in patients with advanced hepatocellular carcinoma from Asia. J Clin Oncol. 2013; 31: 3501-8.

6. Martinez-Balibrea E, Martinez-Cardus A, Gines A, Ruiz de Porras V, Moutinho C, Layos L, et al. Tumor-Related Molecular Mechanisms of Oxaliplatin Resistance. Mol Cancer Ther. 2015; 14: 1767-76.

7. Bu Y, Jia QA, Ren ZG, Zhang JB, Jiang XM, Liang L, et al. Maintenance of stemness in oxaliplatin-resistant hepatocellular carcinoma is associated with increased autocrine of IGF1. PLoS One. 2014; 9: e89686.

8. Zeng Z, Yang L, Ding X, Wang W. Expressions of cysteine-rich61, connective tissue growth factor and Nov genes in hepatocellular carcinoma and their clinical significance. World J Gastroenterol. 2005; 10: 3414-18.

9. Urtasun R, Latasa MU, Demartis MI, Balzani S, Goni S, Garcia-Irigoyen O, et al. Connective tissue growth factor autocriny in human hepatocellular carcinoma: oncogenic role and regulation by epidermal growth factor receptor/yes-associated protein-mediated activation. Hepatology. 2011; 54: 2149-58.

10. Moroishi T, Hansen CG, Guan KL. The emerging roles of YAP and TAZ in cancer. Nat Rev Cancer. 2015; 15: 73-9.

11. Tao Y, Cai F, Shan L, Jiang H, Ma L, Yu Y. The Hippo signaling pathway: an emerging anti-cancer drug target. Discov Med. 2017; 24: 7-18.

12. Zhao $Y$, Yang $X$. The Hippo pathway in chemotherapeutic drug resistance. Int J Cancer. 2015; 137: 2767-73.

13. Wu T, Chen W, Liu S, Lu H, Wang H, Kong D, et al. Huaier suppresses proliferation and induces apoptosis in human pulmonary cancer cells via upregulation ofmiR-26b-5p. FEBS Letters. 2014; 588: 2107-14.

14. Shan L, Li Y, Jiang H, Tao Y, Qian Z, Li L, et al. Huaier Restrains Proliferative and Migratory Potential of Hepatocellular Carcinoma Cells Partially Through Decreased Yes-Associated Protein 1. J Cancer. 2017; 8: 4087-97.

15. Ma L, Wang J, Lin J, Pan Q, Yu Y, Sun F. Cluster of differentiation 166 (CD166) regulated by phosphatidylinositide 3-Kinase (PI3K)/AKT signaling to exert its anti-apoptotic role via yes-associated protein (YAP) in liver cancer. J Biol Chem. 2014; 289: 6921-33.

16. Wang J, Ma L, Weng W, Qiao Y, Zhang Y, He J, et al. Mutual interaction between YAP and CREB promotes tumorigenesis in liver cancer. Hepatology. 2013; 58: 1011-20.

17. Ma L, Cui J, Xi H, Bian S, Wei B, Chen L. Fat4 suppression induces Yap translocation accounting for the promoted proliferation and migration of gastric cancer cells. Cancer Biol Ther. 2016; 17: 36-47.

18. Zhang S, Song X, Cao D, Xu Z, Fan B, Che L, et al. Pan-mTOR inhibitor MLN0128 is effective against intrahepatic cholangiocarcinoma in mice. Journal of Hepatology. 2017; 67: 1194-203.

19. Liu Y, Bi T, Wang Z, Wu G, Qian L, Gao Q, et al. Oxymatrine synergistically enhances antitumor activity of oxaliplatin in colon carcinoma through PI3K/AKT/mTOR pathway. Apoptosis. 2016; 21: 1398-407. 